\title{
IMPROVED CONFINEMENT WITH EDGE RADIATIVE COOLING AT HIGH DENSITIES AND HIGH HEATING POWER IN TEXTOR
}

\author{
A.M. MESSIAEN ${ }^{1 *}$, J. ONGENA ${ }^{1 *}$, U. SAMM ${ }^{2}$, B. UNTERBERG ${ }^{2}$, P.E. VANDENPLAS ${ }^{1}$, \\ G. VAN OOST ${ }^{1}$, G. VAN WASSENHOVE ${ }^{1}$, J. WINTER ${ }^{2}$, D. BOUCHER ${ }^{4}$, P. DUMORTIER ${ }^{1}$, \\ F. DURODIÉ ${ }^{1}$, H.G. ESSER ${ }^{2}$, H. EURINGER ${ }^{2}$, B. GIESEN ${ }^{2}$, E. HINTZ ${ }^{2}$, M. LOCHTER ${ }^{2}$, \\ M.Z. TOKAR ${ }^{2 * *}$, G.H. WOLF ${ }^{2}$, G. FUCHS ${ }^{2}$, D.L. HILLIS ${ }^{3}$, F. HOENEN ${ }^{2}$, P. HÜTTEMANN ${ }^{2}$, \\ R. KOCH ${ }^{1}$, L. KÖNEN ${ }^{2}$, H.R. KOSLOWSKI ${ }^{2}$, A. KRÄMER-FLECKEN ${ }^{2}$, D. PETTIAUX ${ }^{1}$, \\ A. POSPIESZCZYK ${ }^{2}$, B. SCHWEER ${ }^{2}$, H. SOLTWISCH ${ }^{2}$, G. TELESCA ${ }^{2}$, R. UHLEMANN ${ }^{2}$, \\ R. VAN NIEUWENHOVE ${ }^{1 *}$, M. VERVIER ${ }^{1}$, G. WAIDMANN ${ }^{2}$, R.R. WEYNANTS ${ }^{1}$ \\ 1 Laboratoire de physique des plasmas/Laboratorium voor Plasmafysica, \\ Association Euratom-Etat belge/Associatie Euratom-Belgische Staat, \\ Ecole royale militaire/Koninklijke Militaire School, \\ Brussels, Belgium \\ 2 Institut für Plasmaphysik, Forschungszentrum Jülich, \\ Euratom-KFA Association, \\ Jülich, Germany \\ 3 Oak Ridge National Laboratory, \\ Oak Ridge, Tennessee, \\ United States of America \\ 4 ITER Joint Central Team, La Jolla, California, \\ United States of America
}

\begin{abstract}
Improved confinement is achieved on TEXTOR under high power conditions (up to $4 \mathrm{MW}$ of additional heating with NBI-co+ICRH, NBI-co + counter or NBI-co + counter +ICRH) with edge radiative cooling employing silicon or neon as the radiating impurities. It is shown that in quasi-stationary conditions up to $85 \%$ of the input power can be radiated. Such high power fractions offer the possibility of utilizing these techniques to facilitate the power exhaust problem for a tokamak reactor. Discharges with edge radiative cooling exhibit enhanced confinement properties at high densities, e.g. at a central line averaged electron density of $7.5 \times 10^{13} \mathrm{~cm}^{-3}$, an enhancement factor of 1.7 over ITER L89-P confinement scaling is obtained with an edge $q$ value as low as 2.7 . Stable discharges have been obtained even with the $q=$ 2 surface located inside the radiating zone. Furthermore, for radiatively cooled discharges heated with balanced NBIco + counter with or without ICRH, supershot-like peaked electron density profiles, with central density values above 1.0 $\times 10^{14} \mathrm{~cm}^{-3}$, are observed. The present results show that there is no impurity accumulation in the centre and the $\mathrm{Ne}$ and/or $\mathrm{Si}$ concentration is so low that the reactivity of the plasma remains unaffected.
\end{abstract}

\section{INTRODUCTION}

Edge radiative cooling of a tokamak is of considerable importance for a future fusion reactor since it could solve the main difficulties related to the interaction of the plasma with the material wall. A cold, highly radiative boundary distributes most of the power uniformly over the first wall. Structural damage due to high local heat deposition could thus be avoided, and the impurity concentration in the plasma caused by erosion of the wall material could be significantly reduced. The common belief is that high radiation levels lead to plasma detach-

\footnotetext{
* Researcher at NFSR, Belgium.

** Permanent address: Institute for High Temperatures,

Moscow, Russian Federation.
}

ment from the limiter [1] and are therefore linked to reduced energy confinement. It is nevertheless possible to avoid detachment if the power density near the edge is sufficiently high, leading to steep edge temperature and density gradients.

Edge radiative cooling without detachment has been successfully applied on TEXTOR by injection of neon at low and moderate heating powers $\left(\mathrm{H}^{0} \rightarrow \mathrm{D}^{+}\right.$injection, up to a total heating power $P_{\text {tot }}=1.6 \mathrm{MW}$ ) under boronized wall conditions (i.e. by depositing a $100 \mathrm{~nm}$ thick layer consisting of boron or a mixture of boron and carbon on the wall [2]). Up to $95 \%$ of the total heating power was radiated in quasi-stationary conditions [3]. It was found that neon cools the edge and not the centre, and no degradation of the confinement was seen in these discharges even at the highest radiation levels obtained. The 
maximum central chord averaged density in these experiments is limited to $\bar{n}_{\mathrm{e} 0}=5.0 \times 10^{13} \mathrm{~cm}^{-3}$, because of the occurrence of MARFEs (a poloidally asymmetric radiating belt located at the high field side [4]), in contrast to the low power ohmic discharges where the density limit is linked to detachment.

A regime of enhanced confinement has been found on TEXTOR (pump limiter tokamak with circular crosssection) with boronized walls. This regime, called I mode, is characteristic for this machine and is obtained with NBI-co injection or NBI-co injection in combination with ICRH and/or NBI-counter injection [5-7]. Deuterium injection in a $\mathrm{D}(\mathrm{H})$ plasma is the usual operating condition. For the case of balanced NBI-co + counter injection (with or without ICRH), strongly peaked density profiles can be obained which are similar to those observed for the supershot regime found in TFTR [8]. The enhancement factor $f_{\mathrm{H}}$ of the confinement time of these discharges is defined with respect to the $\mathrm{L}$ mode scaling ITER L89-P [9]:

$$
\tau=0.038 B_{\mathfrak{t}}^{0.2} \bar{n}_{\mathrm{e} 0}^{0.1} I_{\mathrm{p}}^{0.85} P_{\mathrm{tot}}^{-0.5} R^{1.2} a^{0.3} \kappa^{0.5} A_{\mathrm{i}}^{0.5}
$$

with the confinement time $\tau$ expressed in seconds, the toroidal magnetic field $B_{\mathrm{t}}$ in teslas, the central line averaged electron density $\bar{n}_{\mathrm{e} 0}$ in $10^{13} \mathrm{~cm}^{-3}$, the plasma current $I_{\mathrm{p}}$ in MA, the total applied heating power $P_{\text {tot }}$ in MW and the large and small plasma radii, $R$ and $a$, respectively, in metres; $\kappa$ is the elongation and $A_{\mathrm{i}}$ the ion atomic mass. This enhancement factor $f_{\mathrm{H}}$ is, on the average, 1.65 for stationary conditions with central line averaged densities $1.4<\bar{n}_{\mathrm{e} 0}<4.3 \times 10^{13} \mathrm{~cm}^{-3}$ and is as good as that obtained for stationary $\mathrm{H}$ modes with edge localized modes in divertor machines [6]. No L-H type transition is observed but the edge density and temperature can be as high as those observed in such $\mathrm{H}$ mode discharges for divertor machines of comparable size. The domain of existence of the I mode is limited by a maximum value of $\beta_{\mathrm{p}}=1.5$ and a maximum value of $\beta_{\mathrm{t}}=2.0 I_{\mathrm{p}} / B_{\mathrm{t}} a$ (with the units $\%(\beta), \operatorname{MA}\left(I_{\mathrm{p}}\right), \mathrm{T}\left(B_{\mathrm{t}}\right)$, $\mathrm{m}(a)$ ), i.e. a Troyon factor $g_{\mathrm{T}}$ of 2.0 . For these I mode discharges with boronized wall conditions, the radiated power fraction $\gamma=P_{\text {rad }} / P_{\text {tot }}$ varies between 0.15 and 0.35 , the radiated power $P_{\text {rad }}$ being mainly due to $\mathrm{O}, \mathrm{C}$ and $\mathrm{B}$ impurity radiation. The enhancement factor $f_{\mathrm{H}}$ decreases generally to about 1.25 when $\bar{n}_{\mathrm{e} 0}=4.0$ $\times 10^{13} \mathrm{~cm}^{-3}$. Above these densities, discharges with a significant value of $f_{\mathrm{H}}$ had not yet been obtained.

In the most recent experiments on TEXTOR we found that it is possible to combine the good confinement results of the I mode with edge radiative cooling; furthermore, the enhancement factor has been improved in the density range around $4.0 \times 10^{13} \mathrm{~cm}^{-3}$, and, most remarkably, the regime of improved confinement has been extended to higher densities $\left(\bar{n}_{\mathrm{e} 0}\right.$ up to $7.5 \times 10^{13} \mathrm{~cm}^{-3}$, with central densities in excess of $10^{14} \mathrm{~cm}^{-3}$ ). At the same time, it has been possible to radiate in quasi-stationary conditions up to more than $85 \%$ of the input heating power without decrease of confinement due to detachment and deleterious effects on plasma reactivity. In these conditions, maximum values for $P_{\text {rad }}$ of about $3.5 \mathrm{MW}$ were obtained which correspond to peak values of about $1 \mathrm{MW} / \mathrm{m}^{3}$ for the radiated power density at the plasma edge.

The edge radiating layer is obtained by means of the impurities neon and/or silicon, which for the present experiments have been used in the following ways:

(a) By siliconization: the wall of TEXTOR is coated with a thin silicon layer $80-100 \mathrm{~mm}$ thick [10]. The presence of $\mathrm{Si}$ in the plasma is due to sputtering. This is evident from the measured sputtering yield under conditions with different edge temperatures [10]. The value of $\gamma=$ $P_{\text {rad }} / P_{\text {tot }}$ varies typically between 50 and $75 \%, \gamma$ depends on the edge density and temperature, which under conditions described in Ref. [11] can vary from 10 to $25 \mathrm{eV}$. (b) By neon injection: the neon gas inlet valve is feedback controlled in order to maintain the brilliance of a Ne VIII line to a preprogrammed level. The pumped limiter ALT II [12] acts as a sink for the neon gas and permits a decrease of the neon concentration. Neon injection has been used in discharges with a boronized wall, and neon is then the main radiating impurity. It has also been used in discharges with a siliconized wall, in which case neon and silicon both contribute to the edge radiation. By adjusting the neon level the radiated power fraction can be varied to up to more than $90 \%$ of the input power, without any indication of $\mathrm{Ne}$ or $\mathrm{Si}$ accumulation, as will be discussed in Section 3. A detailed account of the physics of radiative cooling is given in Ref. [13]. Preliminary results with $\mathrm{Si}$ cooling have been presented in Refs [13, 14].

The confinement results are practically independent of the use of silicon and/or neon in establishing the radiating edge: The density range over which a significant improvement of confinement had previously been observed, with the different heating methods used, is now extended to higher densities. With NBI-co+ICRH, an enhancement factor $f_{\mathrm{H}}=1.7$ is observed for $\bar{n}_{\mathrm{e} 0}=5.0$ $\times 10^{13} \mathrm{~cm}^{-3}$ and a central density of $n_{\mathrm{e} 0}=8.5 \times$ $10^{13} \mathrm{~cm}^{-3}$ at $I_{\mathrm{p}}=350 \mathrm{kA}$, lasting several energy confinement times. For the supershot regime (balanced injection with or without ICRH) the confinement improvement of the TEXTOR I mode can be established at the highest densities ever obtained $\left(\bar{n}_{\mathrm{e} 0}=7.5 \times 10^{13} \mathrm{~cm}^{-3}\right)$ with an 
enhancement factor of up to 1.6 - and this up to the highest plasma currents reached in these experiments, $I_{\mathrm{p}}$ $=485 \mathrm{kA}$, corresponding to $q_{\mathrm{a}} \approx 2.7$. Central densities exceeding $n_{\mathrm{e} 0}=1.0 \times 10^{14} \mathrm{~cm}^{-3}$ are obtained in this regime.

It is important to note that in these high current discharges the position of the $q=2$ surface is approximately 8 to $10 \mathrm{~cm}$ inside the limiter radius (from TRANS [15] simulations) and is situated in the radiation zone which extends up to about $15 \mathrm{~cm}$ inside the limiter radius.

The improvement of confinement on TEXTOR at high radiation and high auxiliary power levels exhibits the general trends already observed in the so-called $\mathrm{Z}$ mode of ISX-B [16], where, by applying neon injection in neutral beam heated plasmas, a confinement improvement of up to 1.8 times $\mathrm{L}$ mode was observed at high density with peaked density profiles and with no appreciable increase of $Z_{\text {eff }}$. Note that neon injection also resulted in improved confinement regimes in ohmic discharges in PLT [17], ASDEX [18] and TEXT-U [19].

(a)


All results pertain to circular discharges ( $a=0.46 \mathrm{~m}$, $R=1.75 \mathrm{~m}$ ) limited by the toroidal pumped limiter ALT II [12]. The toroidal magnetic field, $B_{\mathrm{t}}=2.25 \mathrm{~T}$, and heating powers of $1.5 \mathrm{MW}$ of NBI-co or $2 \times 1.5 \mathrm{MW}$ of balanced NBI-co+counter injection, $50 \mathrm{keV} \mathrm{D} \mathrm{D}^{0} \rightarrow$ $\mathrm{D}^{+}$, and up to $2.2 \mathrm{MW}$ of ICRH, minority heating scheme, $H / D$ ratio $<5 \%$, have been applied in these experiments. Both antennas with and without Faraday screen have been used, with no difference in the results.

The main diagnostics used are: (i) a nine channel HCN interferometer for the electron density; (ii) a nine channel electron cyclotron emission (ECE) radiometer for the electron temperature profile; (iii) an array of ten bolometers for the radiated power profile; (iv) a charge exchange emission spectroscopy (CXS) diagnostic for the ion temperature, monitoring the intensity of a C VI line. Special emphasis is put on spectroscopic diagnostics [20] to measure impurity fluxes in the edge $\left(\mathrm{D}_{\alpha}, \mathrm{Ne}\right.$ I, Ne III, Si I and $\mathrm{Si}$ II lines) and more central radiation (Ne VIII, Si XII). For several discharges the emission profile of an Si XII line is also available.

(b)
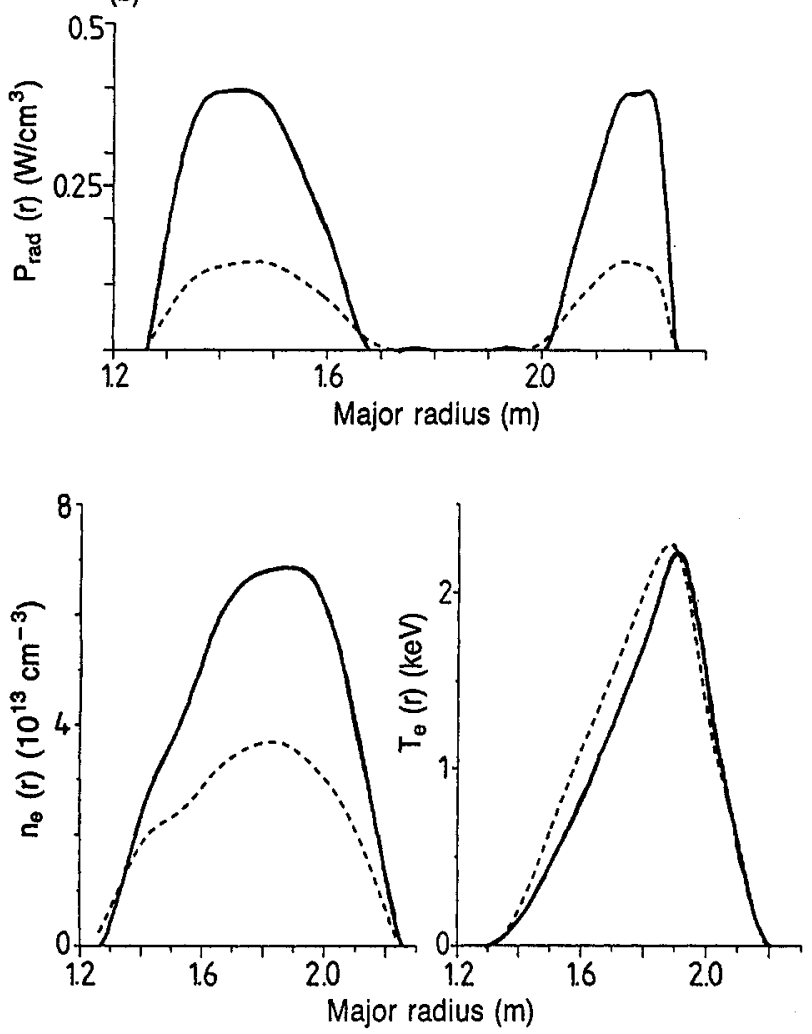

FIG. $1(a)$. Time evolution of diamagnetic and electron energies, $\mathrm{E}_{\text {dia }}$ and $\mathrm{E}_{e}$; central electron temperature, $\mathrm{T}_{e}$; central line averaged electron density, $\overline{\mathrm{n}}_{e 0}$; neutron yield; Ne VIII brilliance; additional heating and radiated power (from bolometry) for a discharge with boronized walls heated by $1.44 \mathrm{MW}$ of NBI-co and $1.63 \mathrm{MW}$ of ICRH with (No. 55232 , solid line) and without (No. 54708 , dashed line) neon cooling at $\mathrm{I}_{p}=350 \mathrm{kA}$. For comparison, we have included the evolution of $\mathrm{E}_{\text {dia }}$ during the neon cooled phase of another discharge with boronized walls (No. 55793 , $\mathrm{P}_{N B t-c o}=1.41 \mathrm{MW}, \mathrm{P}_{I C R H}=1.28 \mathrm{MW}$, dotted line) at a higher current $\left(\mathrm{I}_{p}=480 \mathrm{kA}\right)$. (b) Comparison of radiation profile and electron density and temperature profiles at $\mathrm{t}=2.1 \mathrm{~s}$ for discharges No. 55232 and No. 54708 of Fig. $\mathrm{l}(\mathrm{a})$. 


\section{CONFINEMENT PROPERTIES WITH RADIATIVE COOLING}

\subsection{Results of combined NBI-co injection and ICRH}

Figure 1 shows the main trends of the results corresponding to this heating regime. A discharge heated by 1.6 MW of ICRH and 1.45 MW of NBI-co at a plasma current of $350 \mathrm{kA}$ without radiative cooling (dashed line) is compared to a similar discharge where neon is injected (solid lines). In Fig. 1(a) the following features are shown:

(i) The brilliance of the Ne VIII, which indicates the timing and level of neon injection. The neon gas inlet valve is feedback controlled in order to maintain this brilliance to a preprogrammed level up to $t=2.2 \mathrm{~s}$ for this discharge. After this time the valve is closed and the neon brilliance decays with a time constant of $0.5 \mathrm{~s}$, due to the ALT pumping action.

(ii) The timing and the level of the NBI-co injection and ICRH powers applied. (iii) The central electron temperatures for both discharges, which are the same, even though the central line averaged electron density for the discharge with radiative cooling is much higher. The complete density and electron temperature radial profiles are shown in Fig. 1(b). The peaking factors of the electron density and temperature, defined as the ratio between the peak value and the volume average value of the considered quantity, increase slightly for discharges with radiative cooling. For example, the electron density peaking factor for the discharges shown in Fig. 1(b) changes from 1.7 in the absence of radiation cooling to 1.9 with radiation cooling, and, in the same way, the temperature peaking factor increases from 3.1 to 3.8 .

(iv) The electron and diamagnetic energy, $E_{\mathrm{e}}$ and $E_{\mathrm{dia}}$, for both discharges. The electron energy $E_{\mathrm{e}}$ is nearly equal to half the diamagnetic energy value, which indicates that the contribution of the fast particles to the stored energy is small. With radiative cooling there is no degradation of confinement; on the contrary, the electron and diamagnetic energies for the neon cooled case are main-



FIG. 2. Variation of diamagnetic energy as a function of ICRH power for NBI-co +ICRH heated discharges at $\mathrm{I}_{p}=350 \mathrm{kA}\left(\mathrm{P}_{\text {NBI-co }}=1.45 \mathrm{MW}\right.$ ) with radiative cooling (using $\mathrm{Ne}, \mathrm{Si}$ or both as radiating impurities; indicated by $\mathrm{Ne}$, Si and $\mathrm{Si}+\mathrm{Ne}$ ) or without radiative cooling (boronized walls without neon cooling). Peak and mean stationary values of $\mathrm{E}_{\text {dia }}$ are shown, together with the density domain and the values for the radiated power fraction $\gamma$ corresponding to the different cases. 
tained at a higher level than for a discharge without radiative cooling during the whole heating pulse. This is due to the higher density reached for the same temperature in discharges with radiative cooling (also illustrated in Fig. 1(b)). At the onset of the ICRH pulse, larger values of the stored energy are observed (henceforth to be called peak values), which may last up to $200 \mathrm{~ms}$ (i.e. more than three times the energy confinement time), followed by a decay to a lower value. Sometimes slow oscillations (with a period of a few $100 \mathrm{~ms}$ ) of the stored energy as a function of time are observed. This phenomenon is not yet understood, but the minimum energy value reached in these oscillations remains always larger than the energy values obtained without radiative cooling. Note also that the density varies in a similar way as the stored energy during the heating phase. The behaviour of the stored energy for a radiatively cooled discharge at a higher current $\left(I_{\mathrm{p}}=480 \mathrm{kA}\right)$ is shown by the dotted line for comparison. Its stored energy content is higher (in qualitative agreement with the I mode scaling for which the total stored energy in the discharge is proportional to the plasma current), and the relative amplitude of the oscillations in the stored energy is lower. The variations in stored energy after $t=2.8 \mathrm{~s}$ for $l_{\mathrm{p}}=350 \mathrm{kA}$ and $t=$ $1.8 \mathrm{~s}$ for $I_{\mathrm{p}}=480 \mathrm{kA}$ occur at the ramp-down of the plasma current at the end of the discharge due to the saturation of the iron core of the transformer and could thus be attributed to it.

(v) The neutron yield (to be discussed in Section 3).

(vi) The radiated power. It is much higher in the $\mathrm{Ne}$ cooled discharge $(\gamma=70 \%)$ than in the discharge without radiative cooling $(\gamma=25 \%)$. The profile of the radiated power is shown in Fig. 1(b). Note the presence of the radiating layer at the edge and the absence of radiation at the centre of the discharge.

The effect of edge cooling on the confinement of NBIco +ICRH heated discharges is illustrated in Figs 2 and 3(a). Figure 2 shows the diamagnetic energy values obtained for a constant heating power of $1.45 \mathrm{MW}$ of NBI-co, but at different ICRH power levels, together with the predictions of ITER L89-P, for discharges without radiative cooling (boronized wall) and with a radiating boundary due to neon injection, wall siliconization or to both methods combined. No remarkable difference is seen in the general behaviour of discharges cooled


FIG. 3(a). Density scan of diamagnetic energy for discharges at $\mathrm{I}_{p}=350 \mathrm{kA}$, heated with NBI-co+ICRH $\left(\mathbf{P}_{N B I-c o} \approx 1.45 \mathrm{MW}, \mathrm{P}_{I C R H} \approx 2 \mathrm{MW}\right)$ and balanced injection $\left(\mathrm{P}_{N B /-c o+c o u n t e r} \approx 2.75 \mathrm{MW}\right)$ with and without radiative cooling, together with the predictions of ITER L89-P for the two heating scenarios. Radiative cooling is obtained by neon injection for NBI-co $+I C R H$ heated discharges and by neon injection + siliconized wall for discharges with balanced injection. Data without radiative cooling are obtained in boronized discharges without neon injection. Peak and stationary values observed are indicated for NBI-co $+I C R H$ heated discharges. (b) Enhancement factor $\mathrm{f}_{H}$ with respect to ITER L89-P for diamagnetic energy (peak and stationary values) as a function of central line averaged electron density $\bar{n}_{e 0}$ for NBI-co $+I C R H$ heated discharges at $\mathrm{I}_{p}=350 \mathrm{kA}$, with additional radiative cooling by neon injection. The ICRH power range corresponding to the different points is indicated. 
by neon, silicon or both. In the inset the radiated power fraction seen in these experiments is indicated. Under siliconized conditions, we have $\gamma \approx 60 \%$, whereas, by adjusting the $\mathrm{Ne}$ injection level, $\gamma$ can become as large as $90 \%$. Note that large peak values of the stored energy can already be obtained with moderate ICRH power values; we have indicated in Fig. 2 the peak stored energy values (as observed in Fig. 1, just after the start of ICRH) and the mean values observed after the peak phase during radiative cooling. No large peak stored energy values are present without radiative cooling. We notice that all points corresponding to cooled discharges lie above the stored energy values obtained without radiative cooling and, furthermore, the peak values are observed near the experimental $\beta$ limits of the machine obtained for the I mode regime $[6,7]$. These limits are indicated in the figure and correspond to $152 \mathrm{~kJ}\left(\beta_{\mathrm{p}}=1.5\right)$ and to $149 \mathrm{~kJ}$ (Troyon factor $g_{\mathrm{T}}=2$ ), respectively, for the discharge parameters of Figs 2 and 3(a). The plain symbols in Fig. 3(a) show the variation of the diamagnetic energy with $\bar{n}_{e 0}$ for boronized wall conditions without radiative cooling at a constant level of NBI-co $(\approx 1.45 \mathrm{MW})$ and ICRH ( $\approx 2 \mathrm{MW}$ ), together with the predictions of ITER L89-P for the corresponding power level. The stored energy remains roughly constant with increasing $\bar{n}_{\mathrm{e} 0}$ and has a tendency to decrease at high densities $\left(\bar{n}_{\mathrm{e} 0}>3.5\right.$ $\times 10^{13} \mathrm{~cm}^{-3}$ ); this is clearly seen for the case of balanced NBI-co+counter heating. This results in a decrease of $f_{\mathrm{H}}$ with increasing $\bar{n}_{\mathrm{e} 0}$. For comparison, the open symbols in Fig. 3(a) show the stored energy values reached for the same heating conditions but with radiation cooling. The improvement of the confinement, together with the larger values obtained for $\bar{n}_{\mathrm{e} 0}$ for discharges with radiative cooling, is clear from this figure. Strikingly, if the stored energy or the enhancement factor $f_{\mathrm{H}}$ versus ITER L89-P is plotted as a function of $\bar{n}_{\mathrm{e} 0}$ during the radiative cooling phase, we obtain, in most of the discharges for both peak and stationary phases (dividing the cooling phase into time intervals of $100 \mathrm{~ms}$ and taking for each sample of $100 \mathrm{~ms}$ both the energy and density values), a good correlation between the increase of the stored energy (or the confinement enhancement factor $f_{\mathrm{H}}$ ) and the density. This is shown in Fig. 3(b) for discharges heated with a combination of NBI-co (1.45 MW) and two different ranges of ICRH power. The maximum density values obtained do not necessarily correspond to the maximum values of the additional heating power or the radiated power fraction $\gamma$. This behaviour of $f_{\mathrm{H}}$ versus $\bar{n}_{\mathrm{e} 0}$ is to be compared with the results observed on ISX-B for the so-called $\mathrm{Z}$ mode [16], where the improvement of confinement is also increasing with density. Such a behaviour is not observed in the absence of radiative cooling on TEXTOR; on the contrary, a decrease of the stored energy with increasing densities is obtained. The appearance of large peak stored energy values and of possible stored energy oscillations during the cooling phase is probably linked to this correlation between stored energy and density behaviour. More experimental data are needed to reveal the exact mechanism of this phenomenon.

\subsection{Balanced injection with and without ICRH (supershot-like discharges)}

A high level of radiative cooling, accompanied by a gain in confinement, has also been obtained with pure balanced injection (2.75 MW) as indicated in Fig. 3(a), where a data set without radiative cooling (boronized conditions), which strikingly shows the decrease of confinement at high density, is compared to the results obtained with radiative cooling. The corresponding predictions of the ITER L89-P scaling are also indicated. From the figure it appears that the $f_{\mathrm{H}}$ values obtained for balanced



FIG. 4. Comparison of peak ion and electron temperatures, $\mathbf{T}_{i 0}$ and $\mathrm{T}_{e 0}$ (measured by CXS and ECE diagnostics, respectively), obtained in discharges with and without radiative cooling at $\mathrm{I}_{p}=400 \mathrm{kA}$ and heated with $\approx 1.5 \mathrm{MW}$ of NBI-co and $\approx 1.5 \mathrm{MW}$ of NBI-counter. The data with radiative cooling are obtained in siliconized discharges $(\gamma-60 \%) ;$ the data without radiative cooling are obtained in boronized discharges $(\gamma \approx 25 \%)$. Values for $\mathrm{T}_{e 0}$ at $\overline{\mathrm{n}}_{e 0}>5.0$ $\times 10^{13} \mathrm{~cm}^{-3}$ are not available because of the cut-off of the central ECE channels. 
injection or co-injection + ICRH (stationary values) are roughly the same with radiation cooling. The confinement enhancement is confirmed by measurements of the central plasma temperatures, as illustrated in Fig. 4, showing the increase of the central electron and ion temperatures for a given density in siliconized discharges (where $\gamma \approx$ $60 \%$ ) over discharges with a boronized wall. Central electron temperatures for $\bar{n}_{\mathrm{e} 0}$ above $5.0 \times 10^{13} \mathrm{~cm}^{-3}$ could not be measured because the central ECE channels are at cut-off because of the high central density reached (see, e.g., Fig. 6(a)).

Two examples for discharge behaviour in this heating regime are shown:

First, a comparison is given in Fig. 5(a) between a discharge without radiative cooling (boronized wall) and a discharge with neon and silicon cooling (siliconized wall) heated with balanced NBI-co+counter injection. Note that, notwithstanding the higher density, the siliconized discharge has a larger stored energy than the boronized discharge for a slightly lower input power. The profiles of the radiated power and the electron density and temper-

(a)

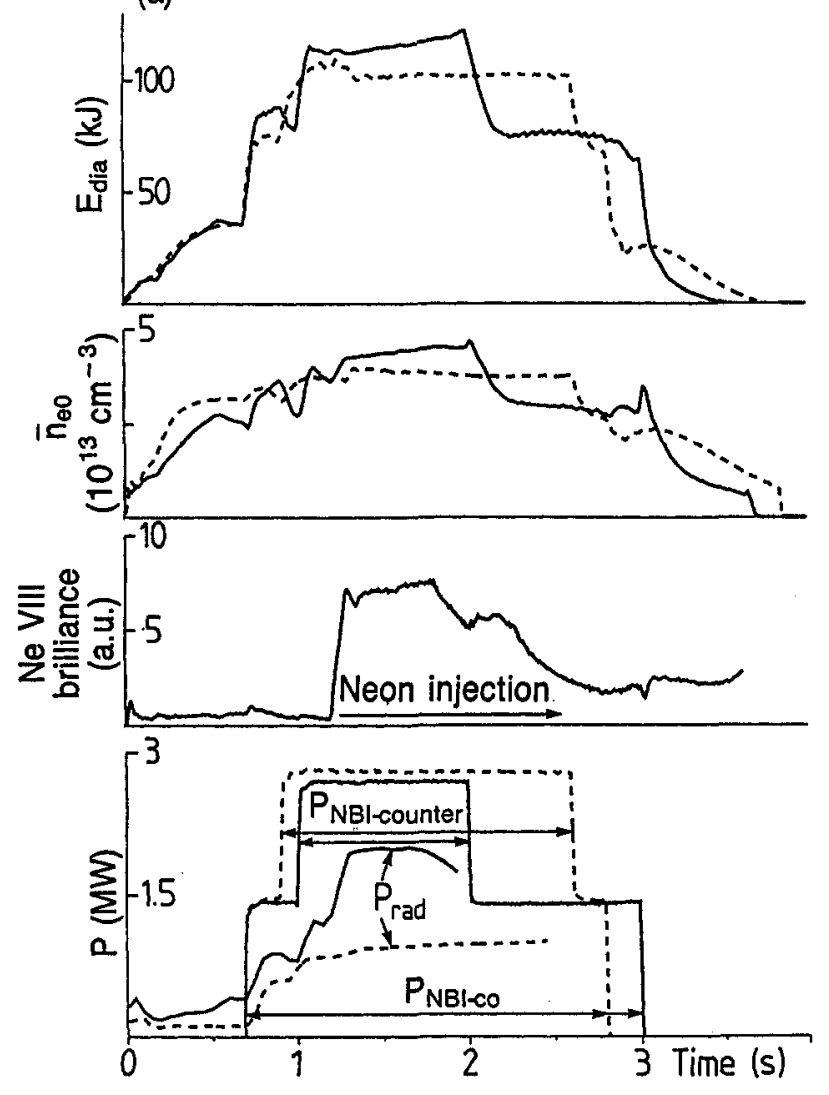

ature for the discharges of Fig. 5(a) are compared in Fig. 5(b). Their behaviour is similar to that observed in discharges heated by combined co-injection and ICRH.

Second, the main characteristics of a discharge with $\mathrm{Si}$ cooling (i.e. with siliconized wall) in which the density was ramped up by deuterium gas puffing are shown in Fig. 6: (i) the feedback controlled electron density evolution as a function of time together with its preprogrammed reference value; (ii) the neutral beam power; (iii) the central ion and electron temperatures, measured by CXS and ECE diagnostics, respectively; the electron temperature can only be measured up to $t=$ $1.6 \mathrm{~s}$, because the central ECE channels are cut off by the high central electron density at $t>1.6 \mathrm{~s}$; (iv) the diamagnetic energy (and electron energy up to $t=1.6 \mathrm{~s}$ ) which stays constant even with increasing density up to the appearance of MHD events leading to a transition to lower confinement levels; (v) the radiation profile which remains unchanged when the density is increased; (vi) the corresponding density profile during the density rise.
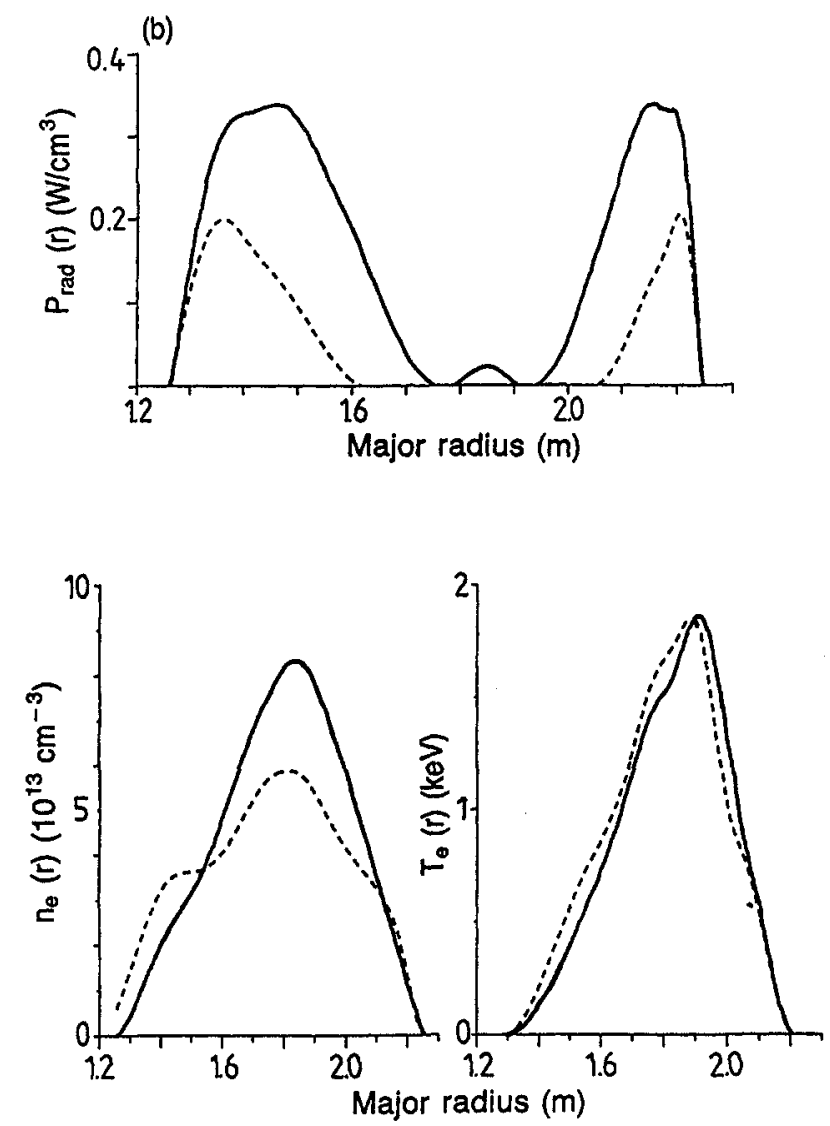

FIG. $5(a)$. Comparison of time evolutions of diamagnetic energy, $\mathrm{E}_{\text {dia; }}$ central line averaged electron density, $\overline{\mathrm{n}}_{e 0} ;$ Ne VIII brilliance; additional heating power $\mathrm{P}_{N B I ;}$ and radiated power $\mathrm{P}_{\text {rad }}$ (from bolometry) for two discharges of the supershot type, heated by balanced NBI-co +counter injection with (No. 56 037, siliconized discharge + neon injection, solid line) and without radiative cooling (No. 54 698, boronized discharge, dashed line) at $\mathrm{I}_{p}=350 \mathrm{kA}$. (b) Radial profiles of radiated power and electron density and temperature at $\mathrm{t}=1.5 \mathrm{~s}$ for the discharges of Fig. 5 (a). 



FIG. 6(a). Time evolution of line averaged density, stored energy, central plasma temperatures and heating powers applied for a discharge (No. 53 040) of the supershot type with silicon cooling where the plasma density is ramped up by a feedback controlled deuterium gas puff. (b) Profiles of density and radiated power at three different times for the same discharge.

It is clear from Fig. 6(b) that the peaked density profile, characteristic for supershot conditions, is maintained with radiative cooling, although for the few cases we have at hand, the shoulders with neon injection are not as well developed as those for the present discharge with Si cooling (siliconized wall) or without edge cooling (see Fig. 5(b)). At the highest densities obtained $\left(\bar{n}_{\mathrm{e} 0}=7.0\right.$ $\left.\times 10^{13} \mathrm{~cm}^{-3}\right)$, central values exceeding $n_{\mathrm{e} 0}=1.0$ $\times 10^{14} \mathrm{~cm}^{-3}$ are reached. Note that the strong peaking of the density profile is still seen at $\bar{n}_{\mathrm{e} 0}>5.5 \times$ $10^{13} \mathrm{~cm}^{-3}$, with a contribution of the beam fuelling to the total particle fuelling rate of less than $10 \%$. In these discharges, there is also no large overshoot of the stored energy such as that observed for NBI-co + ICRH heated discharges, but sometimes a stepwise decay of the stored energy down to the value obtained in the absence of radia- 
tive cooling is observed. These discharges are characterized by the appearance of MHD events which always occur when one of the experimental $\beta$ limits is approached or when high central toroidal $\beta$ values exceeding several per cent are reached (as, e.g., in Fig. 6). The radial profiles of the electron diffusivity computed by TRANSP for such a case are discussed in Ref. [10].

When ICRH is added, a behaviour similar to that of the balanced NBI-co + counter case is seen but with a synergistic increase of the stored energy due to the addition of ICRH [21]. The synergy is seen in the incremental confinement time of the ICRH: compared to an ohmic plasma, a larger incremental ICRH confinement time is found in the presence of neutral beam heating. No large overshoot is seen in the energy at the onset of ICRH. Only if the total amount of heating power produces stored energy values near the $\beta$ limits of the machine does the stored energy drop to levels corresponding to lower values of $f_{\mathrm{H}}$ or even to the $\mathrm{L}$ mode.

Under the best conditions at high plasma currents $\left(I_{\mathrm{p}}=485 \mathrm{kA}, q_{\mathrm{a}}=2.7\right)$ with a total heating power of $4.0 \mathrm{MW}\left(P_{\mathrm{NI}} \sim 2.64 \mathrm{MW}, P_{\mathrm{ICRH}} \approx 1.12 \mathrm{MW}\right)$, a diamagnetic energy of $206 \mathrm{~kJ}$ has been reached with a radiated power level of $2.4 \mathrm{MW}$ for quasi-stationary conditions $\left(\Delta t \approx 400 \mathrm{~ms}\right.$, i.e. $\left.\approx 8 \tau_{\mathrm{E}}\right)$ at a density of $\bar{n}_{\mathrm{e} 0}=$ $7.5 \times 10^{13} \mathrm{~cm}^{-3}$, corresponding to an enhancement factor of $f_{\mathrm{H}}=1.6$ and a Troyon factor of about 2.0, a value which corresponds to the experimental $\beta$ limit of TEXTOR.

The potential for the improvement in confinement resulting from edge radiative cooling can best be summarized quantitatively by underlining that $6.25 \mathrm{MW}$ of combined heating power was needed to reach the $\beta$ limits of TEXTOR in discharges with boronized walls without edge radiative cooling at a plasma current $I_{\mathrm{p}}=520 \mathrm{kA}$ [22], while only $4 \mathrm{MW}$ of power is needed to reach these same $\beta$ limits when edge cooling is applied as indicated in the preceding paragraph.

\section{IMPURITIES AND REACTIVITY}

For experiments with radiative cooling at medium power [13] it was shown that the transport of the radiating impurities is different from that of the background plasma particles. In contrast to the peaked electron and deuterium densities, the impurity density profile does not show any peaking, but a flat or even hollow impurity density profile is inferred from the measurement of the central impurity concentration (always about $1 \%$ ) and the impurity concentration at the edge. This is obtained under quasi- stationary conditions and is based on measurements from CXS, emission spectroscopy in front of the limiter, soft $\mathrm{X}$ ray emission from the core and from conductivity and reactivity analyses.

At high power levels in deuterium discharges heated by deuterium beams, the same set of diagnostic tools is available except for the soft X ray and the CXS impurity concentration diagnostics. Nevertheless, as explained below, there is ample evidence that under improved confinement conditions at high heating powers, there is no significant change in the impurity profiles, keeping the impurity concentration in the centre low:

(a) A first evidence is provided by the profile of the radiated power (Figs $1(b), 5(b)$ and $6(b)$ ), which remains hollow in the centre for the whole heating phase, thus indicating that there is no impurity accumulation in the plasma core. This is confirmed by the brilliance and radial emission profile of the impurity lines (e.g. C V and Si XII), which remain constant during the heating phase. Taking the maximum of the measured values for the central radiation, assuming coronal equilibrium in the centre of the discharge and attributing the central radiation only to the presence of neon, we find about $1 \%$ as an upper limit for the central neon concentration, which is similar to the value found in low power experiments.

(b) An estimate of $Z_{\text {eff }}$ is obtained from TRANSP simulations (on the assumption that the same value prevails at all plasma radii) by imposing the condition that the calculated value of the loop voltage should be equal to the measured value. Simulations of neon cooled discharges heated with NBI-co+ICRH resulted in $Z_{\text {eff }}$ values between 1.5 and 2 . The lowest $Z_{\text {eff }}$ values $\left(Z_{\text {eff }}<\right.$ $1.5)$ have been found under freshly siliconized conditions for discharges heated with balanced injection. When radiative cooling is abruptly started during a discharge heated by balanced injection or balanced injection + ICRH (by imposing a step in the neon radiation), the TRANSP simulation indicates an increase of 0.7 for $Z_{\text {eff }}$, which corresponds to an increase of $0.8 \%$ in the total neon concentration. These numbers for the neon concentration are in agreement with the published results for NBI-co only where concentrations of $0.3 \%$ were quoted for a radiation level of $1 \mathrm{MW}$ [23] and $0.7 \%$ for $1.7 \mathrm{MW}$ of radiated power [3].

(c) The clearest indication of a low central impurity concentration is the absence of a significant decrease of reactivity of the plasma. In the case of deuterium injection in a plasma containing predominantly deuterium on TEXTOR, the neutron yield is mainly due to beam-target reactions [24] for discharges heated by NBI-co or NBI-co + ICRH (hydrogen minority heating scenario). The beam-target reaction rate is approximately indepen- 




FIG. 7. Evolution of neutron yield as a function of central electron temperature during $N B I$-co + ICRH heated discharges with different amounts of radiated power for given NBI conditions and different levels of ICRH power $\left(\mathrm{I}_{p}=350 \mathrm{kA}\right)$.



FIG. 8. Neutron yield as a function of central line averaged electron density $\overline{\mathrm{n}}_{e 0}$, obtained in discharges heated with $\mathrm{NBI}$-co and balanced $\mathrm{NBI}$-co +counter injection, with silicon radiative cooling $(\gamma \approx 60 \%$ ) or without radiative cooling (boronized wall, $\gamma \approx 25 \%$ ).

dent of $n_{\mathrm{e} 0}$, the maximum electron density in the plasma, but scales roughly proportional to $\dot{n}_{0}\left(n_{\mathrm{p} 0} / n_{\mathrm{e} 0}\right) f_{1}\left(T_{\mathrm{e} 0}, E_{\mathrm{b}}\right)$, where $\dot{n}_{0}$ is the number of fast beam neutrals injected per second and the function $f_{1}\left(T_{\mathrm{e} 0}, E_{\mathrm{b}}\right)$ is proportional to $T_{\mathrm{e} 0}$ for the discharges discussed in the present paper (injection energy $E_{\mathrm{b}} \approx 50 \mathrm{keV}$ and central plasma temperature $\left.T_{\mathrm{e} 0} \leq 3 \mathrm{keV}\right)$. Thus the beam-target contribution should decrease in proportion to the central deuterium concentration $n_{\mathrm{D} 0} / n_{\mathrm{e} 0}$ if impurities accumulate in the centre. For the case of balanced injection there is also a significant beam-beam contribution to the neutron production. The beam-beam reaction rate is proportional to $f_{2}\left(T_{\mathrm{e} 0}, E_{\mathrm{b}}\right) / n_{\mathrm{e} 0}^{2}$ and becomes negligible at high densities. Most of the discharges discussed here occur at densities that are high enough for the beam-target reaction to be indeed the predominant neutron production process. 
Simulations by TRANSP predict, at $t=2 \mathrm{~s}$, for discharge No. 55232 heated by NBI-co + ICRH and shown in Fig. 1, a contribution of $89 \%$ of beam-target reactions, of $8.5 \%$ of thermal reactions and of $2.5 \%$ of beam-beam reactions to the total neutron yield. For the discharges with siliconized walls heated with balanced NBI-co + counter, shown in Fig. 8, the corresponding contributions amount, respectively, to 79,4 and $17 \%$ at a density of $\bar{n}_{\mathrm{e} 0}=3.9 \times 10^{13} \mathrm{~cm}^{-3}$ and to 90,2 and $8 \%$ at a density of $\bar{n}_{\mathrm{e} 0}=5.1 \times 10^{13} \mathrm{~cm}^{-3}$.

The neutron yield as a function of $T_{\mathrm{e} 0}$ for discharges heated with NBI-co + ICRH is shown in Fig. 7 for different amounts of neon injected. There is clearly no decrease in reactivity with increasing radiated power fraction $\gamma$ (or, equivalently, with the amount of neon injected), but on the contrary there is even an increase in reactivity at a fixed density, as the central electron temperature increases when neon cooling is applied. This can already be seen in Fig. 1(a), where the reactivity is the same because (i) the electron temperature in both discharges is the same and (ii) the dilution of the deuterium at the discharge centre due to neon injection remains negligible throughout the radiative cooling phase. For the case of discharges with siliconized walls and balanced NBIco + counter injection, the reactivity for $\gamma=60 \%$ is also higher than that for discharges with boronized walls at the same density, as shown in Fig. 8. The higher central electron temperature in the plasma at a given density leads to longer beam slowing-down times and thus to an enhanced neutron production from beam-target and beam-beam reactions. The neutron yield analysis given above indicates thus that there is no significant decrease of reactivity due to a possible central concentration of impurity but that, on the contrary, a higher reactivity is observed in comparison with similar discharges at the same density without radiative cooling which is due to (i) better confinement and resulting higher central temperature and (ii) the absence of significant impurity accumulation at the centre.

(d) In simulations of discharge No. 55232 with neon injection (Fig. 1) using the predictive code PRETOR [25], based on the Rebut-Lallia-Watkins transport model, fair agreement with the measured electron density and temperature profiles, stored energy, total radiated power and loop voltage could only be obtained if a hollow $Z_{\text {eff }}$ profile was assumed, which is again an indirect proof of the absence of impurity accumulation at the centre. When the $Z_{\text {eff }}$ profile was not hollow no simultaneous agreement with all the quantities quoted above could be obtained.

In conclusion, the central concentration of the radiating impurity ( $\mathrm{Ne}$ and/or $\mathrm{Si}$ ) is always about $1 \%$ at the centre of the discharge. The edge concentration inferred from measurements of the neon and deuterium fluxes at the plasma boundary ranges from 2 to $4 \%$. We thus arrive at the same conclusion as for discharges at medium power levels discussed in Ref. [13], i.e. the existence of a flat or somewhat hollow impurity density profile.

\section{DISCUSSION AND CONCLUSIONS}

The reason for the confinement improvement when radiative cooling is applied is not clear. A qualitative explanation has recently been proposed by Kadomtsev in Ref. [26]: the presence of heavier ions in the plasma periphery would decrease the level of turbulent fluctuations at the plasma edge and these, in turn, would less intensively excite microinstabilities in the plasma core. Such a mechanism could be the reason for the confinement improvement by puffing in additional impurities; it is doubtful whether this mechanism could also explain the improved confinement without radiative cooling (I mode). Simulations with the predictive PRETOR code cannot explain the improvement of confinement at the plasma edge since this code uses the measured edge temperatures and densities as boundary conditions. However, the Rebut-Lallia-Watkins energy transport model implemented in the code leads to an improved confinement in the plasma core as a result of steeper current density profiles resulting from the hollow $Z_{\text {eff }}$ profile.

The cause of the small neon or silicon concentration in the plasma centre, leading to a flat or even hollow impurity density profile, is not yet clear. A peaking of the electron density profile is not necessarily related to a peaking of an impurity density profile, as was already mentioned in Section 3. In neoclassical as well as in anomalous transport theories, there are mechanisms which can drive the impurities outwards. In the neoclassical theory, this can occur in the Pfirsch-Schlüter regime if the electron temperature gradient is sufficiently large $[27,28]$. In anomalous transport theories, asymmetric impurity sources can lead to an additional outward drift, as, e.g., shown for ohmic discharges in TEXTOR [29].

To summarize, we have shown that we were able to radiate up to $3.5 \mathrm{MW}$ of power by means of neon injection under improved confinement conditions, with the radiating impurity concentration not affecting the plasma reactivity. In addition, no accumulation of impurities in the centre is observed during the radiatively cooled phase, and this holds for all the heating methods applied. These results thus demonstrate a very promising possibility of solving the problem of energy exhaust and that of good confinement in a reactor at the same time. 
For reactor conditions, calculations assuming noncoronal equilibrium show that the radiation layer extends to about $200-300 \mathrm{eV}$ using $\mathrm{Ne}$ or $\mathrm{Si}$, and that a radiation level is achievable which is of the right order of magnitude to radiate the $\alpha$ power produced. The position of the radiating layer could be adjusted by choosing heavier impurities. The crucial question remains whether the impurity transport will be the same as that observed in the present experiments. It could be smaller, lowering the central impurity concentration and thus allowing an even higher radiation level to be reached by puffing a larger impurity flux in the edge. The opposite could, however, also be true. The study of impurity transport and its experimental verification in larger machines are, therefore, of paramount importance to the extrapolation of the reported results to a reactor.

\section{ACKNOWLEDGEMENTS}

The continuous support by the technical staff of the IPP-KFA and the LPP-ERM/KMS as well as by Dr. Korten's computer division is gratefully acknowledged. The authors would also like to thank Drs R. Goldston, R. Budny and D. McCune of the Princeton Plasma Physics Laboratory for providing them with TRANSP and for their support in using this code system.

\section{REFERENCES}

[1] BUSH, C.E., et al., J, Nucl. Mater. 176\&177 (1990) 786

[2] WINTER, J., et al., J. Nucl. Mater. 162-164 (1989) 713.

[3] SAMM, U., et al., in Plasma Physics and Controlled Nuclear Fusion Research 1992 (Proc. 14th Int. Conf. Würzburg, 1992), Vol. 1, IAEA, Vienna (1993) 309.

[4] LIPSCHUTZ, B., J. Nucl. Mater. 145-147 (1989) 15.

[5] GaIGNEAUX, M., et al., in Controlled Fusion and Plasma Physics (Proc. 18th Eur. Conf. Berlin, 1991), Vol. 15C, Part I, European Physical Society, Geneva (1991) 409.

[6] ONGENA, J., et al., Nucl. Fusion 33 (1993) 283.

[7] ONGENA, J., et al., in Plasma Physics and Controlled Nuclear Fusion Research 1992 (Proc. 14th Int. Conf. Würzburg, 1992), Vol. 1, IAEA, Vienna (1993) 725.

[8] STRAChAN, J.D., et al., Phys. Rev. Lett. 58 (1987) 1004.
[9] YUSHMANOV, P.N., et al., Nucl. Fusion 30 (1990) 1999.

[10] WINTER, J., et al., Phys. Rev, Lett. 71 (1993) 1549.

[11] UNTERBERG, B., et al., in Controlled Fusion and Plasma Physics (Proc. 20th Eur. Conf. Lisbon, 1993), Vol. 17C, Part II, European Physical Society, Geneva (1993) 663.

[12] DIPPEL, K.-H., et al., J. Nucl. Mater. 145-147 (1989) 3.

[13] SAMM, U., et al., Plasma Phys. Control. Fusion 35 (1993) B167.

[14] ONGENA, J., et al., in Controlled Fusion and Plasma Physics (Proc. 20th Eur. Conf. Lisbon, 1993), Vol. 17C, Part I, European Physical Society, Geneva (1993) 127.

[15] HAWRYLUK, R.J., in Physics of Plasmas Close to Thermonuclear Conditions (Proc. Course Varenna, 1979), Vol. 1, CEC, Brussels (1980) 19.

[16] LAZARUS, E.A., et al., Nucl. Fusion 25 (1985) 135.

[17] HINNOV, E., et al., Nucl. Fusion 22 (1982) 325.

[18] BESSENRODT-WEBERPALS, $M$., et al., in Controlled Fusion and Plasma Physics (Proc. 18th Eur. Conf. Berlin, 1991), Vol. 15C, Part I, European Physical Society, Geneva (1991) 389.

[19] KARZHAVIN, Yu.Y., et al., in Controlled Fusion and Plasma Physics (Proc. 20th Eur. Conf. Lisbon, 1993), Vol. 17C, Part I, European Physical Society, Geneva (1993) 135.

[20] POSPIESZCZYK, A., in Atomic and Plasma Material Interaction Processes in Controlled Thermonuclear Fusion (DRAWIN, H.W., JANEV, R.K., Eds), Elsevier Science Publishers, Amsterdam and New York (1993) 213.

[21] MESSIAEN, A.M., et al., in Radio Frequency Power in Plasmas (Proc. 10th Top. Conf. Boston, 1993), AIP Conf. Proc., No. 289, AIP Press, New York (1994) 32.

[22] MESSIAEN, A.M., et al., Plasma Phys. Control. Fusion 35 (1993) A15.

[23] SAMM, U., et al., in Controlled Fusion and Plasma Physics (Proc. 18th Eur. Conf. Berlin, 1991), Vol. 15C, Part III, European Physical Society, Geneva (1991) 157.

[24] VAN WASSENHOVE, G., et al., in Controlled Fusion and Plasma Heating (Proc. 17th Eur. Conf. Amsterdam, 1990), Vol. 14B, Part III, European Physical Society, Geneva (1990) 1040.

[25] BOUCHER, D., REBUT, P.H., in Advances in Simulation and Modelling of Thermonuclear Plasmas (Proc. IAEA Tech. Comm. Mtg Montreal, 1992), IAEA, Vienna (1993) 142.

[26] KADOMTSEV, B.B., in Tokamak Plasma: A Complex Physical System, Plasma Physics Series, IOP Publishing, Bristol and Philadelphia (1992) 108.

[27] FUSSMAN, G., et al., Plasma Phys. Control. Fusion 33 (1991) 1677.

[28] TOKAR', M.Z., Sov. J. Plasma Phys. (Engl. Transl.) 8 (1982) 253.

[29] CLAASSEN, H.-A., et al., J. Nucl. Mater. 176\&177 (1990) 398.

(Manuscript received 11 August 1993

Final manuscript received 11 April 1994) 